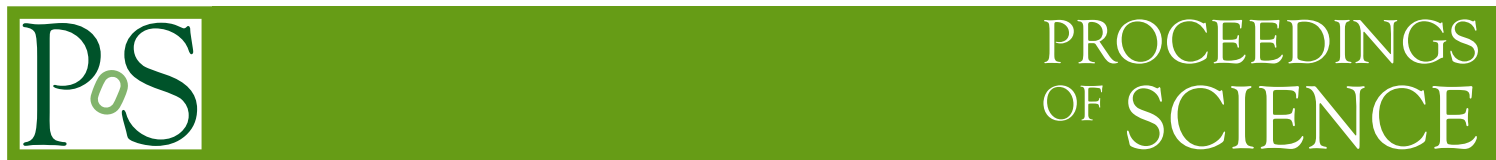

\title{
Formation of the first stars
}

\section{Volker Bromm*}

Department of Astronomy, University of Texas at Austin, U.S.A.

E-mail: vbrommeastro.as.utexas.edu

The first stars, the so-called Population III, fundamentally transformed the early universe at the end of the cosmic dark ages. They provided the first sources of ionizing radiation and heavy elements, thus initiating the evolutionary chain that eventually led to the complex structure we observe today. Based on numerical simulations of the primordial star formation process, it is widely held that the first stars were typically very massive $\left(\sim 10^{2} M_{\odot}\right)$. This standard picture has recently been modified to one where small multiple systems form, instead of just one very massive star. The resulting mass function is still top-heavy, but has an extension to somewhat lower masses as well. Another focus of recent simulations is the assembly of the first galaxies. Their emergence is shaped by the feedback from the Population III stars that had formed and died in the smaller, minihalo, progenitor systems. These early dwarf galaxies are the primary target for upcoming observations with the James Webb Space Telescope.

11th Symposium on Nuclei in the Cosmos

19-23 July 2010

Heidelberg, Germany.

\footnotetext{
${ }^{*}$ Speaker.
} 


\section{Introduction}

An important open question in cosmology is to understand how and when the cosmic dark ages ended [12]. Within the current $\Lambda \mathrm{CDM}$ paradigm, the first stars, the so-called Population III (Pop III), are predicted to have formed at redshifts $z \simeq 20-30$. Their emergence signals the rapid transformation of the universe into an increasingly complex, hierarchical system, due to the energy and heavy element input from Pop III stars and accreting black holes [3, 36, 7, 13]. Currently, we can directly probe the state of the universe 400,000 years after the Big Bang by detecting the temperature anisotropies in the cosmic microwave background (CMB), thus providing us with the initial conditions for subsequent structure formation. Complementary to the CMB observations, we can probe cosmic history all the way from the present-day universe to roughly a billion years after the Big Bang, using the best available ground- and space-based telescopes. In between lies the remaining frontier, and the first stars and galaxies are the sign-posts of this early, formative epoch.

The formation of the very first stars occurred in dark matter minihalos at $z \sim 20-30$. The current standard model posits that Pop III stellar masses were very high, with masses $\sim 100 M_{\odot}$, because gravity needs to be stronger to overwhelm the thermal pressure in the hot primordial gas, where efficient metal coolants are absent [7]. Recently, extremely high-resolution simulations, coupled with sink-particle techniques, have discovered that the first stars typically formed in small stellar groups, possibly dominated by a massive binary $[49,46,16]$. The initial mass function (IMF) of the first stars would then be quite broad, still being dominated by massive stars, but with an extension to lower masses as well. The implications of such possibly widespread binarity for the element production and final fate of Pop III stars still need to be worked out. However, a novel picture of primordial star formation seems to arise, where the process involves the build-up of protostellar disks, gravitational instability in these disks, and a complex dynamics of multiple protostars that interact with the gas in their birth cloud.

To simulate the build-up of the first stellar systems inside high-redshift dwarf galaxies, we have to address the feedback from the first stars on the surrounding intergalactic medium (IGM), and the formation of the second generation of stars out of material that was influenced by this feedback. There are a number of reasons why addressing the feedback from the first stars and understanding second-generation star formation is crucial: (i) The first steps in the hierarchical build-up of structure provide us with a simplified laboratory for studying galaxy formation, which is one of the main outstanding problems in cosmology. (ii) The initial burst of Pop III star formation may have been rather brief due to the strong negative feedback effects that likely acted to self-limit this formation mode $[53,22]$. Second-generation star formation, therefore, might well have been cosmologically dominant compared to Pop III stars. (iii) A subset of second-generation stars, those with masses below $\simeq 1 M_{\odot}$, would have survived to the present day. Surveys of extremely metal-poor Galactic halo stars therefore provide an indirect window into the Pop III era by scrutinizing their chemical abundance patterns, which reflect the enrichment from a single, or at most a small multiple of, Pop III SNe [4, 20, 21, 30]. Stellar archaeology thus provides unique empirical constraints for numerical simulations, from which one can derive theoretical abundance patterns to be compared with the data.

Existing and planned observatories, such as HST, Keck, VLT, and the James Webb Space Telescope (JWST), planned for launch around 2015, yield data on stars and quasars less than a billion 
years after the Big Bang. The ongoing Swift gamma-ray burst (GRB) mission provides us with a possible window into massive star formation at the highest redshifts $[32,8,11]$. This endeavor has already found a GRB at $z \simeq 8.2$, the currently highest redshift known for any object, including galaxies and quasars $[39,48]$. Upcoming missions, such as EXIST and JANUS, are specifically designed to hunt for very high- $z$ bursts, including those from Pop III stars. Measurements of the near-IR cosmic background radiation, both in terms of the spectral energy distribution and the angular fluctuations provide additional constraints on the overall energy production due to the first stars $[40,34,18,31,19]$. Understanding the formation of the first sources of light is thus of great interest to observational studies conducted both at high redshifts and in our local Galactic neighborhood.

\section{Formation of the First Stars}

The first stars in the universe formed a few 100 Myr after the Big Bang, when the primordial gas was first able to cool and collapse into dark matter (DM) minihalos (see Fig. 1) with masses of the order of $10^{6} M_{\odot}[1,6,56,55]$. These stars are believed to have been very massive, with masses of the order of $100 M_{\odot}$, owing to the limited cooling ability of primordial gas in minihalos via the radiation from $\mathrm{H}_{2}$ molecules. Numerical simulations have converged on characterising the properties of the Jeans-unstable cloud in the center of the minihalos, the immediate progenitor for Pop III star formation. The primordial gas settles into a "characteristic state" with $T \sim 200 \mathrm{~K}$ and $n \sim 10^{4} \mathrm{~cm}^{-3}$ [6]. These values for temperature and number density can be understood in terms of the microphysics of the $\mathrm{H}_{2}$ molecule whose rotational transitions provide the relevant cooling mechanism. The characteristic, or "loitering" state is therefore a robust feature of the problem, and most simulations indeed show such behavior. The corresponding Jeans mass is $M_{\mathrm{J}} \sim 10^{3} M_{\odot}$, and once sufficient gas has assembled in the central cloud, so that $M>M_{\mathrm{J}}$, gravitational instability sets in. Before that happens, the gas cloud goes through a slow phase of quasi-hydrostatic evolution, so that a centrally peaked density distribution is established. During this phase, any remaining density fluctuations are wiped out through sound waves. There are then no seeds for any subfragmentation, at least not during the dynamical collapse of the Jeans-unstable cloud.

The initial runaway collapse will lead to the formation of an optically-thick protostellar core. The latter has an initial mass, $\sim 10^{-2} M_{\odot}$, which is very similar to the present-day case [55]. The build-up process depends on the detailed physics of accretion and protostellar feedback. Based on idealized models, it has been argued that the growth of a Pop III star proceeds in a disk-like fashion, again similar to the Pop I situation [47, 35]. Recently, it has become computationally feasible to study the accretion process within the context of realistic cosmological initial conditions [46]. The emerging picture is that of a disk growing around a central dominant protostar until it becomes gravitationally unstable and fragments (see Fig. 2). Other simulations as well have found signs of binary, or small multiple system, formation [49]. Again, the Pop III case might be less different from present-day massive star formation than previously thought. However, the task still remains to couple the 3-dimensional Pop III assembly with the radiation-hydrodynamics of protostellar feedback. It is to be expected that such radiative effects will hinder fragmentation into secondary clumps, once the first few protostars have emerged, and have grown to masses $\sim 10 M_{\odot}$, at which point the hot photosphere is able to emit copious amounts of ionizing UV radiation. The 

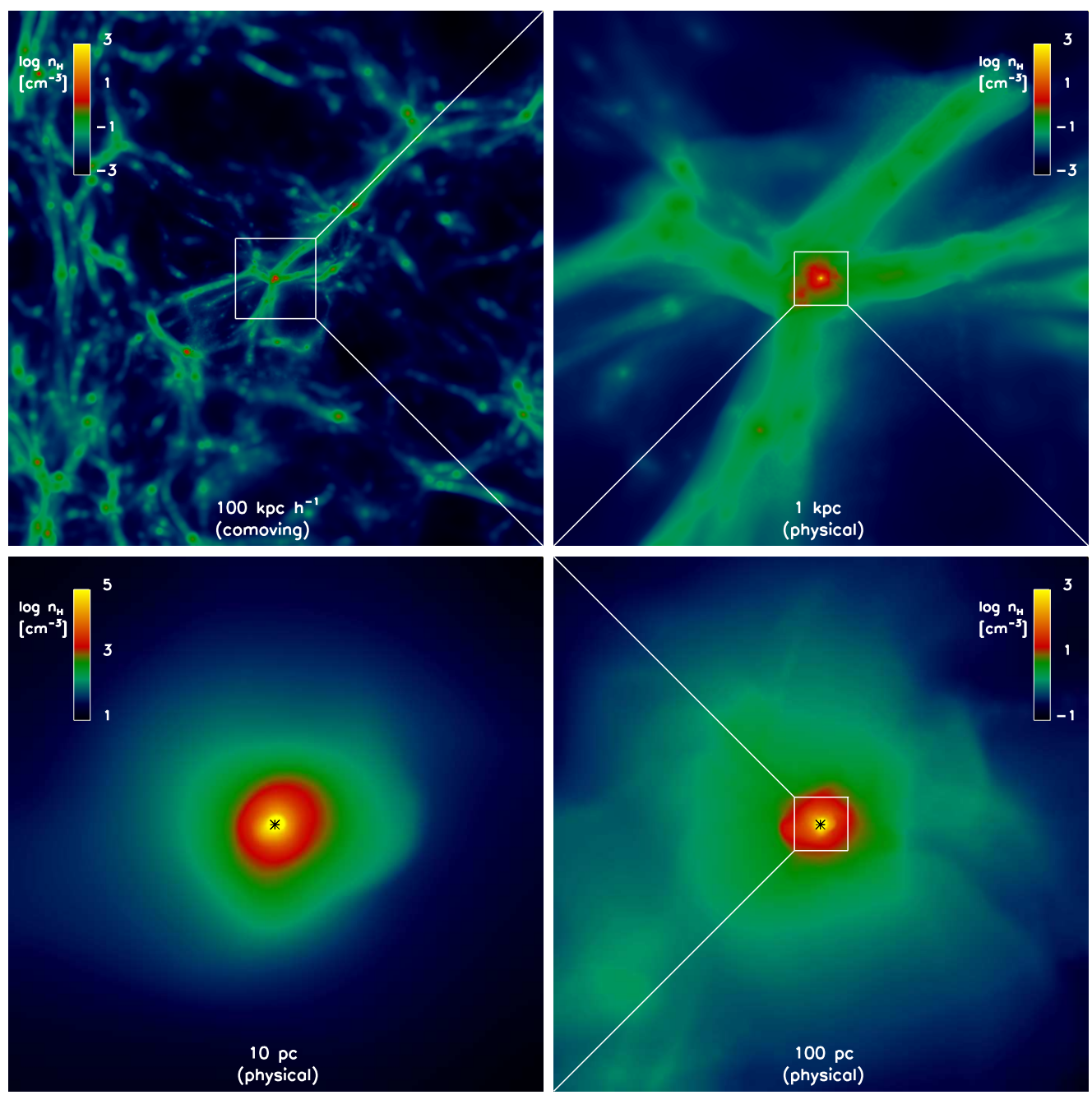

Figure 1: Cosmological environment for Pop III star formation (from [46]). Shown is the gas density distribution on progressively smaller scales, as labeled in each panel. In the bottom two panels, the asterisk denotes the location of the first sink formed, which acts as a proxy for the growing protostar. The top two panels show the emergence of a minihalo, inside of which the dissipating gas assumes a quasi-hydrostatic morphology, just prior to the onset of runaway collapse.

concomitant photo-ionization heating will then raise the temperature in the circumstellar gas to a few $10^{4} \mathrm{~K}$, resulting in a corresponding increase of the Jeans mass.

While the initial conditions for the formation of the very first stars are known from precision measurements of cosmological parameters [44], the situation for the subsequent generations of stars is much more complex. It has become evident that Pop III star formation might actually consist of two distinct modes: one where the primordial gas collapses into a DM minihalo, and one where the metal-free gas becomes significantly ionized prior to the onset of gravitational runaway collapse [27]. To clearly indicate that both modes pertain to metal-free star formation, Chris McKee has suggested a new classification scheme (see [35, 29]). Within this scheme, the minihalo Pop III mode is termed Pop III.1, whereas the second mode is called Pop III.2. 

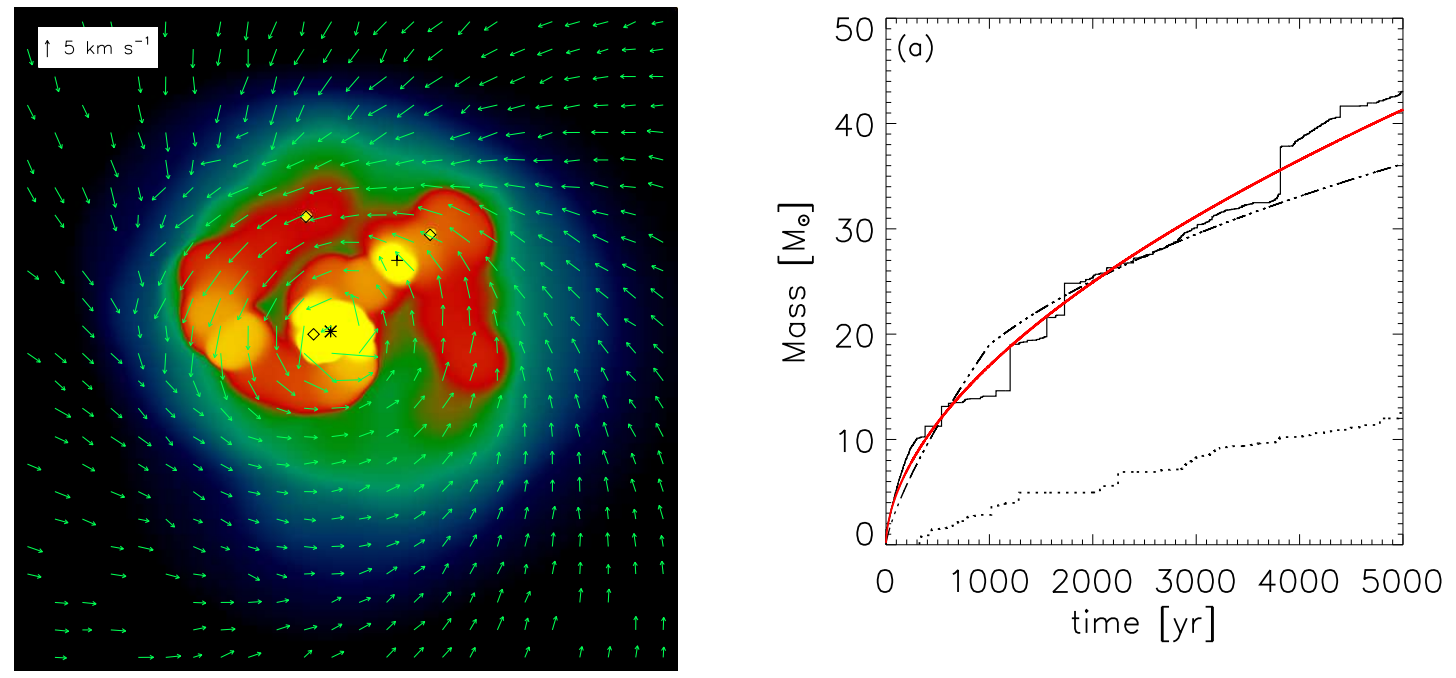

Figure 2: Disk formation and protostellar accretion (from [46]). Left panel: Velocity field of the gas in the center of a minihalo. Sinks are denoted as follows: The asterisk marks the location of the most massive sink, a cross that of the second most massive one, and diamonds represent the other, smaller sinks. Shown is the situation $5000 \mathrm{yr}$ after initial sink formation. At this point, an ordered, nearly Keplerian velocity structure has been established within the disk. Right panel: Sink mass vs. time. The solid line shows the mass of the first sink particle, fitted by a power law according to $M \propto t^{0.55}$ (red line). The dash-dotted line depicts the accretion history found in [10]. The dotted line traces the mass growth of the second largest sink. It is evident that the sinks grow to masses $\gtrsim 10 M_{\odot}$ within a few $1,000 \mathrm{yr}$.

While the formation of the very first, Pop III.1, stars in minihalos relied on $\mathrm{H}_{2}$ cooling, the HD molecule can play an important role in the cooling of primordial gas in several situations, allowing the temperature to drop well below $200 \mathrm{~K}[1,6]$. In turn, this efficient cooling may lead to the formation of primordial stars with masses of the order of $10 M_{\odot}$, the so-called Pop III.2 [27]. In general, the formation of $\mathrm{HD}$, and the concomitant cooling that it provides, is found to occur efficiently in primordial gas which is strongly ionized, owing ultimately to the high abundance of electrons which serve as catalyst for molecule formation in the early universe [42]. Efficient cooling by HD can be triggered within the relic H II regions that surround Pop III.1 stars at the end of their brief lifetimes [2], owing to the high electron fraction that persists in the gas as it cools and recombines $[28,54]$. The efficient formation of HD can also take place when the primordial gas is collisionally ionized, such as behind the shocks driven by the first $\mathrm{SNe}$ or in the virialization of massive DM halos [33, 27, 43].

There might thus be a progression of characteristic masses of the various stellar populations that form in the early universe. In the wake of Pop III.1 stars (typically with $M_{*} \sim 100 M_{\odot}$ ) formed in DM minihalos, Pop III.2 star formation (with $M_{*} \sim 10 M_{\odot}$ ) ensues in regions which have been previously ionized, typically associated with relic H II regions left over from massive Pop III.1 stars collapsing to black holes, while even later, when the primordial gas is locally enriched with metals, Pop II (with $M_{*} \sim 1 M_{\odot}$ ) stars begin to form [9, 22]. Some simulations confirm this picture, as Pop III.2 star formation ensues in relic H II regions in well under a Hubble time, while the formation of Pop II stars after the first SN explosions is delayed by more than a Hubble time 
$[23,57,54,50]$. Other studies argue for a less clear-cut sequence of mass scales [15].

A related question is concerned with the transition from the high-mass dominated Pop III star formation mode to a more normal Pop II one. It is likely that a number of factors play a role in this transition, such as the presence of supersonic turbulence, magnetic fields, and cosmic rays [45]. It is widely believed, however, that the key factor is the onset of heavy-element cooling, leading to the concept of a "critical metallicity", above which low-mass star formation is enabled [9]. The value of this key parameter is the subject of a vigorous debate, but the typical range is $Z_{\text {crit }} \sim 10^{-6}-10^{-3} Z_{\odot}$. The lower value pertains to models where the transition is attributed to dust cooling [41], whereas the higher one arises in models driven by atomic fine-structure lines, in particular of neutral oxygen, and singly ionized carbon. The physics behind the critical metallicity is very complex, so that theorists are in urgent need for empirical guidance. Such hints are indeed provided by Stellar Archaeology, by probing the abundances that are key for cooling in extremely metal-poor halo stars [20]. The evidence so far seems to favor the predictions for threshold abundances derived within the fine-structure driven model.

\section{Formation of the First Galaxies}

How massive were the first galaxies, and when did they emerge? Theory predicts that DM halos containing a mass of $\sim 10^{8} M_{\odot}$ and collapsing at $z \sim 10$ were the hosts for the first bona fide galaxies. These dwarf systems are special in that their associated virial temperature exceeds the threshold, $\sim 10^{4} \mathrm{~K}$, for cooling due to atomic hydrogen [37]. These so-called 'atomic-cooling halos' did not rely on the presence of molecular hydrogen to enable cooling of the primordial gas. In addition, their potential wells were sufficiently deep to retain photoheated gas, in contrast to the shallow potential wells of minihalos [17]. These are arguably minimum requirements to set up a self-regulated process of star formation that comprises more than one generation of stars, and is embedded in a multi-phase interstellar medium.

One of the important consequences of atomic cooling is the softening of the equation of state below the virial radius, allowing a fraction of the potential energy to be converted into kinetic energy $[51,24]$. This implies that perturbations in the gravitational potential can generate turbulent motions on galactic scales, which are then transported to the centre of the galaxy. In this context the distinction between two fundamentally different modes of accretion becomes important [24]. Gas accreted directly from the IGM is heated to the virial temperature and comprises the sole channel of inflow until cooling in filaments becomes important. This mode is termed hot accretion, and dominates in low-mass halos at high redshift. The formation of the virial shock and the concomitant heating in an atomic cooling halo are visible in Fig. 3. The second mode, termed cold accretion, becomes important as soon as filaments are massive enough to enable molecule reformation, which allows the gas to cool and flow into the central regions of the nascent galaxy with high velocities. Although the assembly of the first galaxies provides us with an idealized laboratory for galaxy formation in general, the degree of complexity that is exhibited in the corresponding merger tree is already considerable. Still, current supercomputer simulations have just reached the capacity to address the first galaxy formation process in an a-priori fashion, one star at a time.

Cold accretion is a viable agent for driving turbulence, due to the large amount of kinetic energy it brings to the center of the galaxy. Two physically distinct mechanisms are responsible 


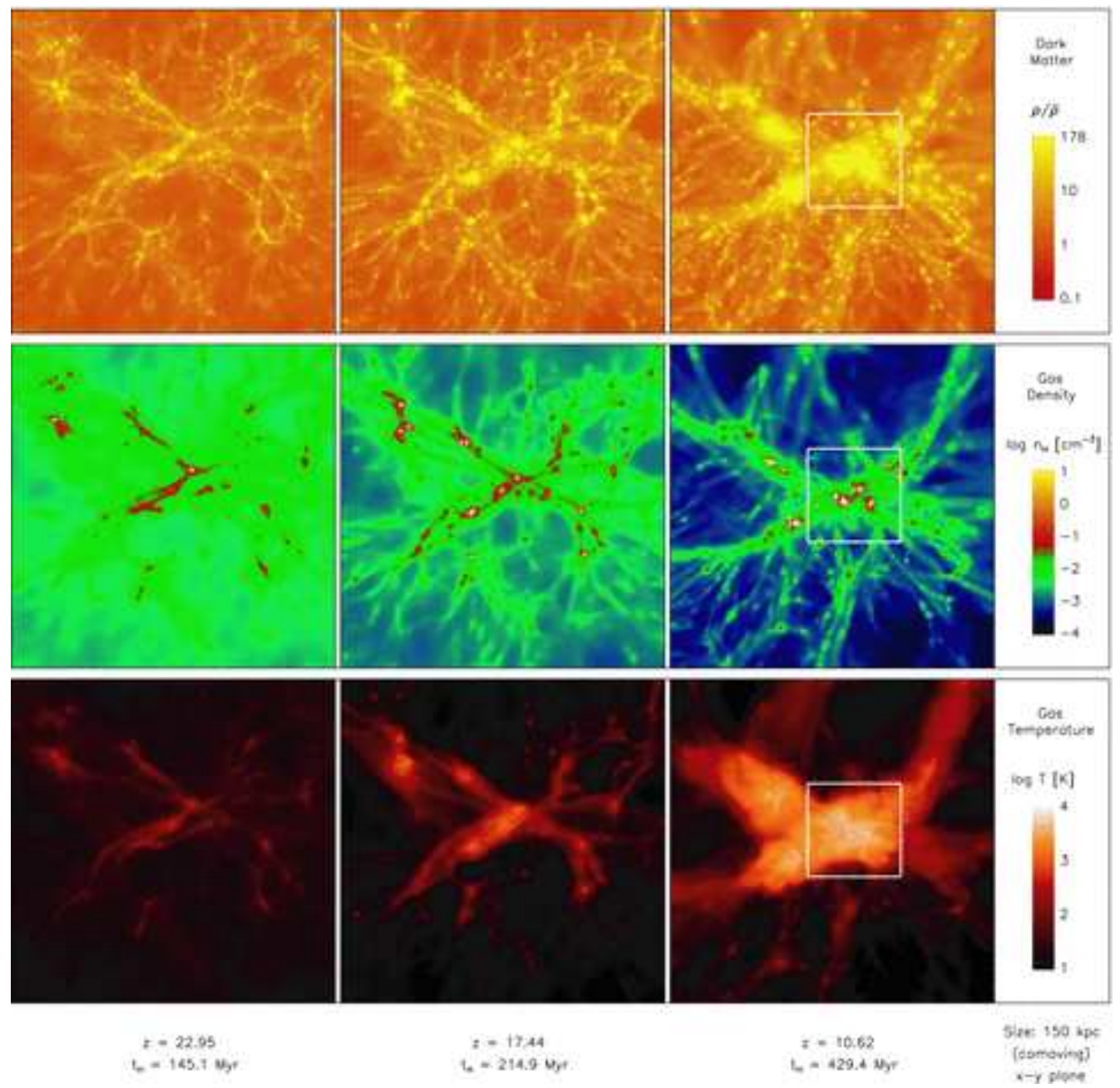

Figure 3: Assembly of the first galaxy (from [24]). Gravity is assembling the dark matter (top row) and pristine gas (middle row) into a primordial galaxy, here taken to be an atomic cooling halo. Shown is the evolution at three different stages (from left to right), from the time where the first minihalo triggers Pop III star formation, to the virialization of the atomic cooling halo at $z \simeq 10$. White crosses indicate individual Pop III stars, which form prior to the first galaxy. The assembly process is accompanied by powerful virialization shocks that heat up the infalling gas to $>10^{4} \mathrm{~K}$ (bottom row). Most of the gas that ends up in the central cloud, about to undergo a powerful starburst, was funneled in via cold accretion streams along the cosmological filaments.

for creating shocks [24]. The virial shock forms where the ratio of infall velocity to local sound speed approaches unity, while a multitude of unorganized shocks forms near the center of the galaxy and is mostly caused by accretion of cold, high-velocity gas from filaments. These are more pronounced than the virial shock and have a significantly higher angular component. They create transitory density perturbations that could in principle become Jeans-unstable and trigger the gravitational collapse of individual clumps. In concert with metal enrichment by previous star formation in minihalos, metal mixing in the first galaxies will likely be highly efficient and could lead to the formation of the first low-mass star clusters [14, 38], in extreme cases possibly even 
to metal-poor globular clusters [5]. Some of the extremely iron-deficient, but carbon and oxygenenhanced stars observed in the halo of the Milky Way may thus have formed as early as redshift $z \simeq 10[30]$.

The first galaxies were also the likely site for the formation of the first low-mass stars [21]. If only one of the progenitor minihalos that merged into an atomic cooling halo hosted a massive Pop III star, capable of exploding either as a PISN or core-collapse hypernova [26], the central gas in the newly virialized atomic cooling halo would already be enriched to levels of $Z \sim 10^{-3} Z_{\odot}$ $[52,25]$. The subsequent starburst would then give rise to Pop II stars, some of them surviving to the present where stellar archaeological surveys, such as SEGUE [4], can probe them. At this junction, we encounter a number of computational challenges. To fully describe the initial conditions for the second-generation starburst, we need to consider cases where multiple SN explosions contribute to the enrichment. We then need to accurately follow the turbulent mixing of the enriched gas on its way from the minihalo, through the IGM, and back into the virializing first galaxy, often assumed to be identical with atomic cooling halos. We finally need to simulate the collapse and fragmentation of the central gas cloud that will result in a Pop II cluster. An interesting question in this context is whether some of these clusters can already reach masses that would qualify them as globular clusters [5]. We expect to have at least preliminary answers to these problems within the next few years, thanks in large part to the availability of large-scale supercomputing facilities.

\section{Outlook}

Understanding the formation of the first sources of light marks the frontier of high-redshift structure formation. It is crucial to predict their properties in order to develop the optimal search and survey strategies for the JWST. Whereas ab-initio simulations of the very first stars can be carried out from first principles, and with virtually no free parameters, one faces a much more daunting challenge with the first galaxies. Now, the previous history of star formation has to be considered, leading to enhanced complexity in the assembly of the first galaxies. One by one, all the complex astrophysical processes that play a role in more recent galaxy formation appear back on the scene. Among them are external radiation fields, comprising UV and X-ray photons, and possibly cosmic rays produced in the wake of the first SNe. There will be metal-enriched pockets of gas which could be pervaded by dynamically non-negligible magnetic fields, together with turbulent velocity fields built up during the virialization process. However, the goal of making useful predictions for the first stars and galaxies is now clearly drawing within reach, and the pace of progress is likely to be rapid. A few years from now we should have a better idea of whether our theoretical ideas are on track, or need to be refined.

\section{Acknowledgments}

I would like to thank the organizers for putting together a stimulating program at the intersection of many key challenges in astrophysics. Support from NSF grant AST-0708795 and NASA ATFP grants NNX08AL43G and NNX09AJ33G is gratefully acknowledged. The simulations presented here were carried out at the Texas Advanced Computing Center (TACC). 


\section{References}

[1] T. Abel, G. L. Bryan and M. L. Norman, Science, 295, 93 (2002).

[2] M. A. Alvarez, V. Bromm and P. R. Shapiro, Astrophys. J., 639, 621 (2006).

[3] R. Barkana and A. Loeb, Phys. Rep., 349, 125 (2001).

[4] T. C. Beers and N. Christlieb, Annu. Rev. Astron. Astrophys., 43, 531 (2005).

[5] V. Bromm and C. J. Clarke, Astrophys. J., 566, L1 (2002).

[6] V. Bromm, P. S. Coppi and R. B. Larson, Astrophys. J., 564, 23 (2002).

[7] V. Bromm and R. B. Larson, Annu. Rev. Astron. Astrophys., 42, 79 (2004).

[8] V. Bromm and A. Loeb, Astrophys. J., 575, 111 (2002).

[9] -, Nature, 425, 812 (2003).

[10] —, New Astron., 9, 353 (2004).

[11] —, Astrophys. J., 642, 382 (2006).

[12] V. Bromm, N. Yoshida, L. Hernquist and C. F. McKee, Nature, 459, 49 (2009).

[13] B. Ciardi and A. Ferrara, Space Science Rev., 116, 625 (2005).

[14] P. C. Clark, S. C. O. Glover and R. S. Klessen, Astrophys. J., 672, 757 (2008).

[15] P. C. Clark, S. C. O. Glover, R. S. Klessen and V. Bromm, Astrophys. J., 727, 110 (2011).

[16] P. C. Clark, S. C. O. Glover, R. J. Smith, T. H. Greif, R. S. Klessen and V. Bromm, Science, in press (arXiv:1101.5284) (2011).

[17] M. Dijkstra, Z. Haiman, M. J. Rees and D. H. Weinberg, Astrophys. J., 601, 666 (2004).

[18] E. Dwek, R. G. Arendt and F. Krennrich, Astrophys. J., 635, 784 (2005).

[19] E. R. Fernandez and E. Komatsu, Astrophys. J., 646, 703 (2006).

[20] A. Frebel, J. L. Johnson and V. Bromm, Mon. Not. R. Astron. Soc., 380, L40 (2007).

[21] —, Mon. Not. R. Astron. Soc., 392, L50 (2009).

[22] T. H. Greif and V. Bromm, Mon. Not. R. Astron. Soc., 373, 128 (2006).

[23] T. H. Greif, J. L. Johnson, V. Bromm and R. S. Klessen, Astrophys. J., 670, 1 (2007).

[24] T. H. Greif, J. L. Johnson, R. S. Klessen and V. Bromm, Mon. Not. R. Astron. Soc., 387, 1021 (2008).

[25] T. H. Greif, S. C. O. Glover, V. Bromm and R. S. Klessen, Mon. Not. R. Astron. Soc., 387, 1021 (2010).

[26] N. Iwamoto, H. Umeda, N. Tominaga, K. Nomoto and K. Maeda, Science, 309, 451 (2005).

[27] J. L. Johnson and V. Bromm, Mon. Not. R. Astron. Soc., 366, 247 (2006).

[28] J. L. Johnson, T. H. Greif and V. Bromm, Astrophys. J., 665, 85 (2007).

[29] J. L. Johnson, T. H. Greif and V. Bromm, Mon. Not. R. Astron. Soc., 388, 26 (2008).

[30] T. Karlsson, J. L. Johnson and V. Bromm, Astrophys. J., 679, 6 (2008).

[31] A. Kashlinsky, R. G. Arendt, J. Mather and S. H. Moseley, Nature, 438, 45 (2005). 
[32] D. Q. Lamb and D. E. Reichart, Astrophys. J., 536, 1 (2000).

[33] M. N. Machida, K. Tomisaka, F. Nakamura and M. Y. Fujimoto, Astrophys. J., 622, 39 (2005).

[34] M. Magliocchetti, R. Salvaterra and A. Ferrara, Mon. Not. R. Astron. Soc., 342, L25 (2003).

[35] C. F. McKee and J. C. Tan, Astrophys. J., 681, 771 (2008).

[36] J. Miralda-Escudé, Science, 300, 1904 (2003).

[37] S. P. Oh and Z. Haiman, Astrophys. J., 569, 558 (2002).

[38] C. Safranek-Shrader, V. Bromm and M. Milosavljevic, Astrophys. J., 723, 1568 (2010).

[39] R. Salvaterra et al., Nature, 461, 1258 (2009).

[40] M. R. Santos, V. Bromm and M. Kamionkowski, Mon. Not. R. Astron. Soc., 336, 1082 (2002).

[41] R. Schneider, K. Omukai, A. K. Inoue and A. Ferrara, Mon. Not. R. Astron. Soc., 369, 1437 (2006).

[42] P. R. Shapiro and H. Kang, Astrophys. J., 318, 32 (1987).

[43] Y.-A. Shchekinov and E. O. Vasiliev, Mon. Not. R. Astron. Soc., 368, 454 (2006).

[44] D. N. Spergel et al., Astrophys. J. Supp., 170, 377 (2007).

[45] A. Stacy and V. Bromm, Mon. Not. R. Astron. Soc., 382, 229 (2007).

[46] A. Stacy, T. H. Greif and V. Bromm, Mon. Not. R. Astron. Soc., 403, 45 (2010).

[47] J. C. Tan and C. F. McKee, Astrophys. J., 603, 383 (2004).

[48] N. R. Tanvir et al., Nature, 461, 1254 (2009).

[49] M. J. Turk, T. Abel and B. W. O’Shea, Science, 325, 601 (2009).

[50] D, Whalen, B. van Veelen, B. W. O'Shea and M. L. Norman, Astrophys. J., 682, 49 (2008).

[51] J. H. Wise and T. Abel, Astrophys. J., 665, 899 (2007).

[52] —, Astrophys. J., 685, 40 (2008).

[53] N. Yoshida, V. Bromm and L. Hernquist, Astrophys. J., 605, 579 (2004).

[54] N. Yoshida, K. Omukai and L. Hernquist, Astrophys. J., 667, L117 (2007).

[55] —, Science, 321, 669 (2008).

[56] N. Yoshida, K. Omukai, L. Hernquist and T. Abel, 2006, Astrophys. J., 652, 6 (2006).

[57] N. Yoshida, S. P. Oh, T. Kitayama and L. Hernquist, Astrophys. J., 663, 687 (2007). 